\title{
Preservação da audição residual em pacientes usuários de implante coclear multicanal: estudo piloto
}

\section{Hearing conservation after cochlear implant pillot study}

M. Valéria Schmidt Goffi Gomez ${ }^{1}$, Mariana Cardoso Guedes ${ }^{2}$, Cristina Gomes de Ornelas 2 Sandra Barreto Giorgi Sant'Anna ${ }^{3}$, Rubens Vuono de Brito Neto ${ }^{4}$, Tanit Ganz Sanchez ${ }^{4}$ Ricardo Ferreira Bento

\section{Resumo / Summary}

ntrodução: Com o progresso tecnológico dos implantes cocleares, cada vez mais pacientes com audição residual útil são candidatos em potencial ao implante (Hodges et al., 1997). Apesar disso, não se sabe ao certo se a introdução dos eletrodos poderia destruir as estruturas auditivas envolvidas nessa audição residual. Objetivo: O objetivo deste estudo foi verificar a conservação da audição residual em pacientes usuários de implante coclear multicanal, comparando a audiometria pré e pós-operatória desses indivíduos. Forma de estudo: Clínico prospectivo randomizado. Material e método: Foram estudados 09 pacientes adultos com disacusia sensorioneural bilateral severa a profunda, usuários de Implante coclear Nucleus 22 e 1 paciente usuário de Implante Combi 40+. Resultado: No grupo estudado a conservação auditiva ocorreu em 50\% dos indivíduos, quando considerado o critério de conservação de duas ou mais frequências médias (da fala) e em 80\%, se for considerada audição também em frequências graves. Conclusão: A média de queda dos limiares tonais antes e após a cirurgia foi de $4 \mathrm{~dB}$ na orelha implantada e $0 \mathrm{~dB}$ na orelha contralateral.

\author{
Palavras-chave: preservação da audição, \\ audição residual, implante coclear. \\ Key words: hearing preservation, \\ residual hearing, cochlear implant.
}

ntroduction: Technological development of Cochlear Implants allowed patients with residual hearing, that's to say, severe sensorineural hearing losses, to be considered as candidates (Hodges et al., 1997). Nevertheless, it is not clear whether the electrode array insertion may destroy remaining auditory structures. Aim: The purpose of this research was to verify the maitainance of residual hearing in multichannel cochlear implant users, by the comparison between the pre and post pure tone audiometry. Study design: Clinical prospective randomized. Material and method: Nine Nucleus 22 device and one Combi 40+ device users were evaluated. Results: In this sample hearing preservation was observed in $50 \%$ of the subjects, when considering the mid frequencies (speech frequencies) criterium of preservation. On the other hand, when the whole range of frequencies (including low frequencies) was considered as a criterium the preservation was revealed in $80 \%$ of the subjects. Conclusion: The average decrement in pure tone thresholds was $4 \mathrm{~dB}$ in the implanted ear and 0 $\mathrm{dB}$ in the contralateral ear.

${ }^{1}$ Fonoaudióloga do Setor de Fonoaudiologia da Divisão de Clínica Otorrinolaringológica do HCFMUSP, Doutora em Ciências dos Distúrbios da Comunicação Humana pela UNIFESP/EPM. ${ }^{2}$ Fonoaudióloga Clínica.

${ }^{3}$ Fonoaudióloga Clínica. Mestranda em Fisiopatologia Experimental pela FMUSP.

${ }^{4}$ Médico(a) Assistente da Divisão de Clínica Otorrinolaringológica do Hospital das Clínicas da FMUSP. Doutor(a) em Otorrinolaringologia pela FMUSP. ${ }^{5}$ Professor Associado da Disciplina de Otorrinolaringologia da FMUSP

Trabalho realizado na Divisão de Clínica Otorrinolaringológica do Hospital das Clínicas da Faculdade de Medicina da Universidade de São Paulo. Endereço para correspondência: A/C Maria Valéria S. Goffi Gomez - Av. Dr. Enéas Carvalho de Aguiar 255 São Paulo Clínica Otorrinolaringológica do HCFMUSP 6 ํ Andar - ICHC - 05403-000 Tel. (0xx11) 3069.6288 - E-mail: goffigomez@uol.com.br

Artigo recebido em 9 de agosto de 2001. Artigo aceito em 29 de agosto de 2002. 


\section{INTRODUÇÃO}

Com o progresso tecnológico dos implantes cocleares, cada vez mais pacientes com audição residual útil são candidatos em potencial ao implante ${ }^{1}$.

A indicação do implante coclear tem se extendido a adultos com perdas auditivas severas ${ }^{2,3,4}$, o que tem aumentado o número de indivíduos com bons resíduos auditivos na audiometria pré-operatória.

Cada vez mais acredita-se que indivíduos com maior audição residual teriam melhor aproveitamento do implante coclear $^{1,5}$. Em um estudo com ossos temporais de 13 pacientes que apresentaram perdas auditivas severas, Incesulu \& Nadol (1998) ${ }^{6}$ encontraram maior número de células no gânglio espiral das orelhas com melhor audição residual. Isso poderia implicar em melhor desempenho quanto ao reconhecimento de fala com o implante coclear nessas orelhas.

Apesar disso, não se sabe ao certo se a introdução dos eletrodos poderia destruir as estruturas auditivas envolvidas nessa audição residual. Boggess et al. (1989) ${ }^{7}$ mostraram que a conservação da audição é possível em cerca de 30\% dos indivíduos implantados, porém com redução significativa do limiar na orelha operada.

Em um estudo histopatológico, Jackler et al. (1989)8 observaram a formação de tecido de granulação em volta da janela redonda e inflamação da escala timpânica após a inserção de eletrodos longos em cobaias. Clark et al. (1988) ${ }^{9}$ mostraram que a introdução desses eletrodos causam traumas mínimos nas estruturas cocleares. Na tentativa de posicionar os eletrodos de maneira a estimular os elementos neurais o mais próximo possível, estes são inseridos na escala vestibular ou na escala timpânica usando timpanotomia posterior $^{10}$

A preservação da audição residual após o implante coclear poderia sugerir que tanto a cirurgia como a presença dos eletrodos não influenciam na percepção da orelha operada. Atualmente o conceito de cirurgia conservadora para os implantes cocleares ${ }^{11,12}$ é animador no sentido de não deteriorar a audição residual dos pacientes. Porém ainda persiste a idéia de que a inserção dos eletrodos seja traumática ${ }^{13}$ e que com isso haja perda de células ganglionares remanescentes.

Dessa maneira, a presente pesquisa tem como objetivo verificar a conservação da audição residual em pacientes usuários de implante coclear multicanal, comparando a audiometria pré e pós-operatória desses indivíduos.

\section{MATERIAL E MÉTODO}

Foram implantados em 29 pacientes no Departamento de Clínica Otorrinolaringológica do Hospital das Clínicas FMUSP desde 1999. Desses pacientes, 15 eram do sexo feminino e 14 eram do sexo masculino, com idade variando entre 02 e 77 anos. Desses, apenas um indivíduo não apresentava audição residual em nenhuma das orelhas (cofose).

Tanto a audiometria pré-operatória como a pósoperatória foi realizada utilizando-se o audiômetro Madsen modelo Midimate 622 com saída máxima de $120 \mathrm{~dB}$ para as freqüências de $500 \mathrm{~Hz}$ a $4000 \mathrm{~Hz}$, de $105 \mathrm{~dB}$ para as freqüências de $250 \mathrm{~Hz}$ e $8000 \mathrm{~Hz}$ e de $115 \mathrm{~dB}$ para $6000 \mathrm{~Hz}$. Além da audiometria tonal, realizada de $250 \mathrm{~Hz}$ a $8000 \mathrm{~Hz}$, a avaliação incluiu a pesquisa do limiar de reconhecimento e fala (SRT), quando possível, e do limiar de detecção de fala (SDT). No caso das crianças a audiometria foi realizada através da técnica de condicionamento com encaixes ou com reforço visual, além da observação das respostas comportamentais. Todos os pacientes realizaram também a imitanciometria e testes eletrofisiológicos (audiometria de tronco cerebral BERA e emissões otoacústicas) como parte da avaliação préoperatória.

Desses pacientes, 25 receberam o implante coclear Nucleus 22, 03 receberam o implante Nucleus 24 e um recebeu o implante coclear Combi $40+$.

Para a comparação dos resultados audiológicos pré e pós-operatórios foram obtidas as médias dos limiares tonais (MLT) nas freqüências de $250 \mathrm{~Hz}$ a $2000 \mathrm{~Hz}$ e nas freqüências agudas ( $4 \mathrm{KHz}$ a $8 \mathrm{KHz}$ ). A orelha não operada foi tomada como controle.

\section{RESULTADOS}

Até o momento foram comparados os resultados da audiometria pré e pós-operatória de dez (10) pacientes com idade variando de 12 a 73 anos (média de 56 anos), sendo 04 do sexo feminino e 06 do sexo masculino. A média do tempo de uso do implante coclear foi de 9,6 meses (Tabela 1).

A média dos limiares tonais (MLT) pré-operatório nas freqüências de $250 \mathrm{~Hz}$ a $2 \mathrm{KHz}$ foi de $110,50 \mathrm{~dB}$ na orelha implantada e de $110,05 \mathrm{~dB}$ na orelha controle. Alguns pacientes $(n=03)$ apresentavam audição conservada também nas freqüências agudas, assim a MLT para essa região ( $4 \mathrm{kHz}$ a $8 \mathrm{kHz}$ ) foi de $97,5 \mathrm{~dB}$ em ambas as orelhas.

Na avaliação pós-operatória, a média dos limiares $(250 \mathrm{~Hz}-2000 \mathrm{~Hz})$ caiu $4,72 \mathrm{~dB}$ na orelha implantada e $0,20 \mathrm{~dB}$ na orelha controle. Já a média dos limiares tonais nas freqüências agudas diminuiu $17,5 \mathrm{~dB}$ na orelha implantada e 8,33dB na orelha contralateral (Tabelas 2 e 3).

O Gráfico 1 mostra a variação dos limiares tonais em ambas orelhas para as freqüências de $250 \mathrm{~Hz}$ a $2 \mathrm{KHz}$ e de $4 \mathrm{KHz}$ a $8 \mathrm{KHz}$ :

A conservação da audição na orelha operada (presença de resposta em pelo menos duas das 
Tabela 1. Dados demográficos do grupo estudado

\begin{tabular}{|c|c|c|c|c|c|c|c|}
\hline Paciente & Sexo & Idade & Etiologia & $\begin{array}{c}\text { Tempo de uso } \\
\text { do IC }\end{array}$ & Modelo & $\begin{array}{c}\text { Orelha } \\
\text { Implantada }\end{array}$ & $\begin{array}{l}\text { Eletrodos } \\
\text { Inseridos }\end{array}$ \\
\hline 01 & $M$ & 44 & Congênita & 05 meses & $\mathrm{N} 22$ & OD & Todos \\
\hline 02 & $M$ & 16 & A esclarecer & 07 meses & N22 & OE & Todos \\
\hline 03 & $\mathrm{~F}$ & 66 & Otosclerose & 06 meses & N22 & OE & Todos \\
\hline 04 & $\mathrm{~F}$ & 56 & A esclarecer & 08 meses & N22 & OD & Todos \\
\hline 05 & $M$ & 12 & Congênita & 04 meses & Combi $40+$ & OD & Todos \\
\hline 06 & $\mathrm{~F}$ & 44 & Meningite & 22 meses & N22 & OD & Todos \\
\hline 07 & $M$ & 44 & Meningite & 24 meses & N22 & OD & Todos \\
\hline 08 & $\mathrm{~F}$ & 35 & A esclarecer & 08 meses & $\mathrm{N} 22$ & OD & Todos \\
\hline 09 & $M$ & 19 & A esclarecer & 09 meses & N22 & OD & Todos \\
\hline 10 & $M$ & 73 & Meningite & 03 meses & N22 & OE & Todos \\
\hline
\end{tabular}

Tabela 2. Média dos limiares Tonais $(250 \mathrm{~Hz}-2000 \mathrm{~Hz})$ Pré e Pós-Operatório em todos os pacientes avaliados.

\begin{tabular}{|c|c|c|c|c|c|c|}
\hline \multirow[t]{2}{*}{ Paciente } & \multicolumn{2}{|c|}{ Orelha Implantada } & \multirow[t]{2}{*}{ Diferença } & \multicolumn{2}{|c|}{ Orelha Controle } & \multirow[t]{2}{*}{ Diferença } \\
\hline & MLT Pré & MLT Pós & & MLT Pré & MLT Pós & \\
\hline 01 & 115 & 120 & -5 & 113 & 115 & -2 \\
\hline 02 & 95 & 110 & -15 & 92,5 & 92,5 & 0 \\
\hline 03 & 111,25 & 112,5 & $-1,25$ & 112,5 & 111,25 & $1,25(+)$ \\
\hline 04 & 121,25 & 121,25 & 0 & 121,25 & 121,25 & 0 \\
\hline 05 & 102,5 & 103,75 & $-1,25$ & 100 & 95 & $5(+)$ \\
\hline 06 & 112,5 & 118,75 & $-6,25$ & 120 & 117,5 & $2,5(+)$ \\
\hline 07 & 117,5 & 121,25 & $-3,75$ & 115 & 121,25 & $-6,25$ \\
\hline 08 & 116,25 & 113,5 & $2,75(+)$ & 117,5 & 112,5 & $5(+)$ \\
\hline 09 & 103,75 & 110 & $-6,25$ & 103,75 & 106,25 & $-2,5$ \\
\hline 10 & 110 & 121,25 & $-11,25$ & 105 & 110 & -5 \\
\hline Média & 110,50 & 115,23 & $-4,72$ & 110,05 & 110,25 & $-0,20$ \\
\hline
\end{tabular}

MLT = média dos limiares tonais

Tabela 3. Número de indivíduos com presença de audição nas freqüências agudas e as respectivas médias dos limiares tonais $(4 \mathrm{kHz}-8 \mathrm{kHz})$ Pré e Pós-Operatório.

\begin{tabular}{ccccccc}
\hline$N$ & \multicolumn{2}{c}{ Orelha Implantada } & Diferença & \multicolumn{2}{c}{ Orelha Controle } & Diferença \\
\hline & MLT Pré & MLT Pós & & MLT Pré & 90 & MLT Pré \\
01 & 90 & 112,5 & $-22,5$ & 90 & $-7,5$ & $-1,5$ \\
01 & 105 & 117,5 & $-12,5$ & 102,5 & 115 & -5 \\
09 & $\downarrow$ (ausente) & $\downarrow$ (ausente) & - & 100 & 105 & $-8,33$ \\
Média & 97,5 & 115 & $-17,5$ & 97,5 & 105,83 & -83 \\
\hline
\end{tabular}

MLT = média dos limiares tonais

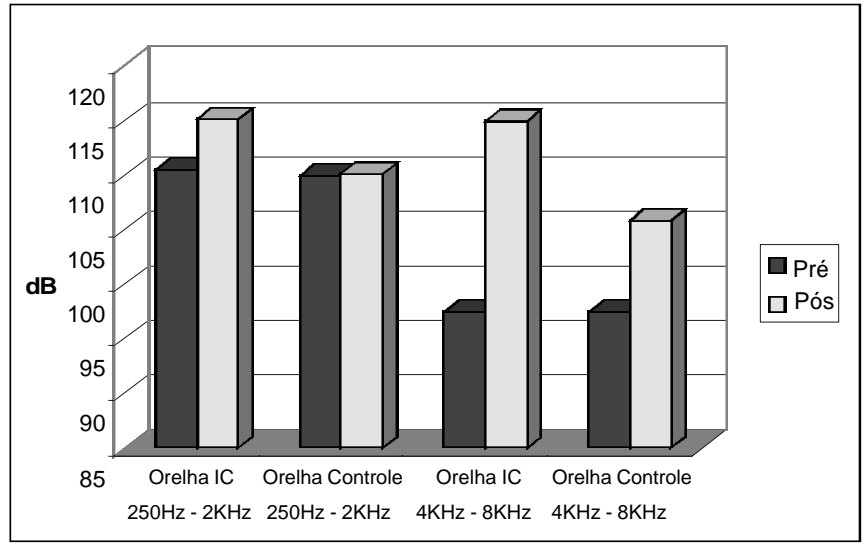

Gráfico 1. Média dos Limiares Tonais Pré e Pós Implante Coclear freqüências da fala) foi observada em 05 pacientes (50\%), sendo que 02 apresentaram respostas nas quatro freqüências $(250,500,1000$ e $2000 \mathrm{~Hz})$. Dos dez pacientes estudados, 05 (50\%) permaneceram com limiares entre $80 \mathrm{~dB}$ e $100 \mathrm{~dB}$ em uma das freqüências da fala na orelha implantada, 02 (20\%) com limiares acima de $100 \mathrm{~dB}$ e 03 (30\%) apresentaram ausência de resposta nas freqüências da fala.

\section{DISCUSSÃO}

Acredita-se que candidatos ao uso do implante coclear com melhores resíduos auditivos teriam resultados com um maior aproveitamento. Entretanto, a escolha da orelha a ser 
implantada é baseada, em muitos casos, no lado com menor restos na tentativa cautelosa de preservar o lado melhor para eventual amplificação. Dessa forma, a conservação da audição estaria clinicamente relacionada com um benefício funcional, ou seja, as habilidades auditivas poderiam ser ampliadas com o uso do implante coclear. Além disso, outro ponto muito discutido é a utilização de amplificação sonora na orelha que foi operada ${ }^{1}$.

No grupo estudado a conservação auditiva ocorreu em 50\% dos indivíduos, quando considerado o critério de conservação de frequências médias (da fala) e em 80\%, se for considerada audição também em frequências graves. A média dos limiares tonais nas freqüências da fala $(250 \mathrm{~Hz}$ a $2000 \mathrm{~Hz}$ ) aumentou em 4,60dB na orelha implantada e em $0,08 \mathrm{~dB}$ na orelha contralateral (controle). Já, nas freqüências agudas ( $4 \mathrm{KHz}$ a $8 \mathrm{KHz}$ ) esse aumento foi de $17,5 \mathrm{~dB}$ na orelha operada e de $8,33 \mathrm{~dB}$ na orelha controle.

Hodges et al. (1997) encontraram aumento do limiar tonal de $12 \mathrm{~dB}$ NA na orelha implantada e de $4,4 \mathrm{~dB}$ NA na orelha contralateral; considerando entretanto a \% de indivíduos com audição conservada mensurável observaram que 52\% (21:40) dos indivíduos apresentaram conservação da audição.

Nesta pesquisa consideramos a freqüência de $250 \mathrm{~Hz}$ como uma freqüência da fala, pois, de acordo com Redondo \& Lopes Fo (1997) ${ }^{14}$, há uma grande importância das freqüências mais graves no reconhecimento de palavras na língua portuguesa. Além disso, optou-se por considerar como preservação da audição a presença de limiar em duas ou mais freqüências entre $250 \mathrm{~Hz}$ e $2 \mathrm{KHz}$ a fim de se evitar respostas de vibração ou "sensação de pressão", comuns em indivíduos com perdas auditivas de grau profundo.

Os mecanismos da perda auditiva causada pelo implante coclear ainda não estão bem definidos. Como observamos, houve uma variação muito maior nas freqüências agudas, o que pode sugerir maior trauma na região basal da cóclea durante a inserção dos eletrodos ${ }^{12}$. Gstoettner et al. (1997) ${ }^{10}$ acreditam que quando a inserção é limitada ao primeiro ponto de resistência os resultados histopatológicos da região apical da cóclea mostrar trauma mínimo. Em outro estudo histopatológico após a implantação de eletrodos longos, Jackler et al. (1989) ${ }^{8}$ observaram degeneração na região basal do órgão de Corti de algumas cobaias. Boggess et al. (1989) 7 acreditam que a perda auditiva após o implante possa estar associada à perda de perilinfa, perda de tecido coclear ou como efeito da vibração mecânica do eletrodo ou da própria estimulação elétrica, rompimento da membrana basilar ou lesão do ligamento espiral (Nadol, 1997) ${ }^{13}$.

Alguns pacientes $(n=04)$ melhoraram o limiar em algumas freqüências, mesmo na orelha implantada. Isso pode ter ocorrido devido à maior atenção ao som e melhora na percepção auditiva proporcionada pelo uso do implante coclear ${ }^{15}$.
Após uma intervenção cirúrgica, mudanças de até $10 \mathrm{~dB}$ nos limiares geralmente são consideradas clinicamente não significativas (Hodges, 1997) ${ }^{1}$. Isso se deve às limitações inerentes ao teste (variações como posicionamento de fones, nível de ruído ambiental e atenção do paciente, por exemplo). Assim, as respostas podem não só piorar, mas também melhorar.

Dessa maneira, podemos considerar que houve preservação da audição após a inserção dos eletrodos nas freqüências mais importantes para a inteligibilidade de fala $(250 \mathrm{~Hz}-2 \mathrm{KHz})$. Kiefer et al. (1998) ${ }^{15}$ estudaram 17 adultos e 18 crianças com audição residual pré-implante e observaram preservação parcial da audição dos adultos. Por outro lado, a audição nas crianças foi preservada. Afastou-se a hipótese de que a deterioração da audição nos adultos fosse devido à progressão da etiologia da perda, considerando-se os resultados inalterados na orelha contralateral.

Não foram analisados dados como a idade e o tempo de estimulação devido ao pequeno número de pacientes. Contudo, estamos dando continuidade a essa pesquisa a fim de obtermos os dados dos demais pacientes implantados no Departamento e uma análise longitudinal da performance desses indivíduos.

Vale ressaltar que, mesmo aqueles que não apresentaram audição residual na audiometria têm boa percepção nos testes funcionais realizados de rotina.

\section{CONCLUSÃO}

1. A preservação da audição pode ser possível com o uso do implante coclear multicanal.

2. A variação dos limiares após a inserção dos eletrodos não é clinicamente significativa.

3. A variação dos limiares é maior nas freqüências agudas do que nas freqüências da fala $(250 \mathrm{~Hz}-2 \mathrm{kHz})$.

\section{REFERÊNCIASBIBUOGRÁFICAS}

1. Hodges AV, Schloffman J, Balkany T. Conservation of Residual Hearing with Cochlear Implantation. The American Journal of Otology 1997;18:179-83.

2. Mangabeira Albernaz PL. Implante coclear. Parte 2. RBM-ORL 1996; 3(2):119-22.

3. Costa Filho OA, Bevilacqua MC, Moret ALM. Critérios de seleção de crianças candidatas ao implante coclear do Hospital de Pesquisa e Reabilitação de Lesões Lábio - Palatais - USP Rev Bras Otorrinolaring 1996;62(4):306-13.

4. Bento RF, Sanchez TG, Brito Neto RV. Critérios de Indicação de Implante Coclear. @ rquivos da FORL 1997a;1(2):66-7.

5. Shiomi Y, Naito Y, Honjo I, Fujiki N, Kaneko K, Takahashi H, Yamashita M, Kawano M. Cochlear Implant in Patients with Residual Hearing. Auris Nasus Larynx 1999 Oct;26(4):369-74.

6. Incesulu A \& Nadol JB Jr. Correlation of Acoustic Thereshold Measures and Spiral Ganglion Cell in Severe to Profound Sensorioneural Hearing Loss: Implications for Cochlear Implantation. Ann Otol Rhinol Laryngol 1998 Nov;107(11Pt 1):906-11. 
7. Boggess W, Baker J, Balkany T. Loss of Residual Hearing After Cochlear Implantation. Laryngoscope 1989;99:102-5.

8. Jackler RK, Leake PA, Mckerrow WS. Cochlear Implantation Revision: Effects of Reimplantation on the Cochlea. Ann Otol Rhinol Laryngol 1989;98:813-9.

9. Clark GM, Shepherd RK, Dowell RC. Histopathological Following Cochlear Implantation in a Patient. Acta Otolaryngol Suppl (Stockh) 1988;106:448.

10. Gstoettner W, Franz P, Hamzavi J, Plenk Jr H, Baumgartner W, Czerny C. Intracochlear position of cochlear implant electrodes. Acta Otolaryngol (Stockh) 1999;119:229-33.

11. Bento RF, Sanchez TG, Brito Neto RV. O Implante FMUSP 1. Técnica Cirúrgica. Arquivos da FORL 1997b;1(3):80-3.
12. Cohen HL. Cochlear implant soft surgery: Fact or fantasy. Otolaryngol Head Neck Surg 1997;117:214-6.

13. Nadol JB. Patterns of neural degeneration in the human cochlea and auditory nerve: implications for cochlear implantation. Otolaryngol Head Neck Surg 1997;117:220-8.

14. Redondo MC \& Lopes Fo O. Testes Básicos de Avaliação Auditiva. In: Lopes Filho O. Tratado de Fonoaudiologia. 1a edição. São Paulo: Ed. Roca; 1997.

15. Kiefer J, Von Ilberg C, Reimer B, Knecht R, Gall V, Diller G, Stürzebecher E, Pfennigdorff T, Spelsberg A. Results of Cochlear Implantation in Patients with severe to profound Hearing loss implications for patient selection. Audiology 1998;37:383-95. 